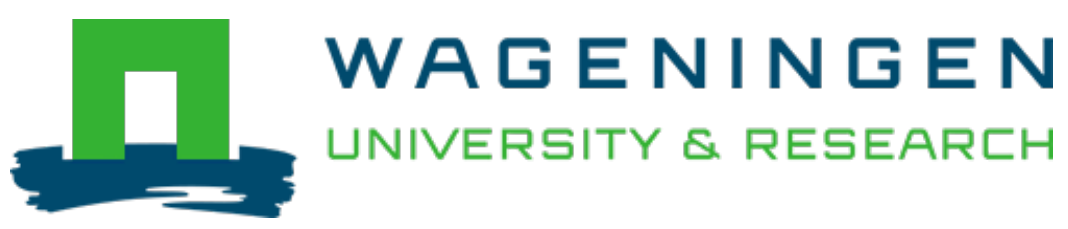

\title{
Formation of Taste-Active Pyridinium Betaine Derivatives Is Promoted in Thermally Treated Oil-in-Water Emulsions and Alkaline pH
}

\author{
Journal of Agricultural and Food Chemistry \\ Troise, Antonio Dario; Berton-Carabin, Claire C.; Vitaglione, Paola; Fogliano, Vincenzo \\ https://doi.org/10.1021/acs.jafc.0c01446
}

This article is made publicly available in the institutional repository of Wageningen University and Research, under the terms of article $25 \mathrm{fa}$ of the Dutch Copyright Act, also known as the Amendment Taverne. This has been done with explicit consent by the author.

Article 25 fa states that the author of a short scientific work funded either wholly or partially by Dutch public funds is entitled to make that work publicly available for no consideration following a reasonable period of time after the work was first published, provided that clear reference is made to the source of the first publication of the work.

This publication is distributed under The Association of Universities in the Netherlands (VSNU) 'Article $25 \mathrm{fa}$ implementation' project. In this project research outputs of researchers employed by Dutch Universities that comply with the legal requirements of Article $25 \mathrm{fa}$ of the Dutch Copyright Act are distributed online and free of cost or other barriers in institutional repositories. Research outputs are distributed six months after their first online publication in the original published version and with proper attribution to the source of the original publication.

You are permitted to download and use the publication for personal purposes. All rights remain with the author(s) and / or copyright owner(s) of this work. Any use of the publication or parts of it other than authorised under article $25 \mathrm{fa}$ of the Dutch Copyright act is prohibited. Wageningen University \& Research and the author(s) of this publication shall not be held responsible or liable for any damages resulting from your (re)use of this publication.

For questions regarding the public availability of this article please contact openscience.library@,wur.nl 


\title{
Formation of Taste-Active Pyridinium Betaine Derivatives Is Promoted in Thermally Treated Oil-in-Water Emulsions and Alkaline $\mathrm{pH}$
}

\author{
Antonio Dario Troise,* Claire C. Berton-Carabin, Paola Vitaglione, and Vincenzo Fogliano \\ Cite This: J. Agric. Food Chem. 2020, 68, 5180-5188 \\ Read Online
}

ACCESS | Lill Metrics \& More | 国 Article Recommendations ｜（s Supporting Information

ABSTRACT: The oil-water interface can be used as an efficient reaction controller in foods by carrying specific reactants and products in either the hydrophobic or hydrophilic phase. The formation of the taste-active compounds $\mathrm{N}$-(1-carboxyethyl)-6hydroxymethyl-pyridinium-3-ol inner salt (alapyridaine) and 1-(1-carboxyethyl)-3-hydroxy-pyridinium inner salt is influenced by the presence of a dispersed saturated triglyceride oil phase and by the $\mathrm{pH}$ of the aqueous phase. At $\mathrm{pH} 6.5$, the formation of both betaines was 1.24 and 6 times higher in emulsions than in aqueous solution after 4 min at $140{ }^{\circ} \mathrm{C}$. In alkaline emulsions $(\mathrm{pH}=9.5,4$ $\mathrm{min})$, the concentrations of alapyridaine and 1-(1-carboxyethyl)-3-hydroxy-pyridinium ion were 6.2 and 3.8 times higher, respectively, than in unbuffered emulsions as a result of the interaction between the polar head group of the surfactant and pyridinium rings. Here, we reported for the first time the effects of multiphase systems on the formation of nonvolatile, taste-active end products.

KEYWORDS: Maillard reaction, Amadori products, mass spectrometry, emulsions, taste-active molecules

\section{INTRODUCTION}

In foods, thermal treatments induce chemical modifications and boost the formation of aroma and taste-active compounds. ${ }^{1}$ Along with lipid oxidation, the Maillard reaction (MR) contributes to the formation of distinct molecules that determine food quality with several implications to physiological aspects. ${ }^{2}$ The concentration and activity of glycation compounds are deeply influenced by the food matrix: the molecular crowding and the actual possibility of precursors to react with each other can be major drivers for the definition of the reaction routes. ${ }^{3}$

The concept of "chemical pools" was introduced by Yaylayan to help probing the reaction network occurring during the $\mathrm{MR}^{4}$ When dealing with food processing, the concept of chemical pools can be useful to describe the relationship between flavors and food structures: the formation of MR intermediates and end products is a consequence of food architecture, particularly, in the case of multiphase dispersed systems. ${ }^{5}$ The chemical nature of the precursors influences the location of such compounds, and according to their partition coefficient, the formation of end products can be modulated through a multicompartmentalization approach of the MR. ${ }^{6}$

In emulsions, partitioning is favored by environments with specific properties and polarities; segregation of the reactants and products can generate unique reaction routes. ${ }^{7}$ Indeed, in oil-in-water $(\mathrm{O} / \mathrm{W})$ emulsions, the large surface area of dispersed droplets facilitates the interactions between lipids and water-soluble compounds, and the total extent of the reaction is modulated by multiple factors such as the $\mathrm{pH}$, particle size, concentration, physical state, oxygen, and light. ${ }^{8}$ An $\mathrm{O} / \mathrm{W}$ emulsion system can be divided into three regions among which nonvolatile molecules distribute themselves: the interior of the nonpolar droplets, aqueous phase, and $\mathrm{O} / \mathrm{W}$ interfacial region. The relative concentration of the target molecules in each of these regions depends on their molecular structure and the properties of each of the phases. ${ }^{9}$ Therefore, the presence of emulsifiers, such as surfactants, which is needed to physically stabilize $\mathrm{O} / \mathrm{W}$ emulsions, may modulate the partitioning of precursors and intermediates, affecting the quantity and kind of end products formed, as previously demonstrated for antioxidants and lipid oxidation products. ${ }^{10}$

The structural organization of food systems offers an interesting perspective to evaluate the effects of the MR cascade in emulsions toward the formation of taste-active molecules. ${ }^{11}$ According to the kind of precursors, the reactivity of amino acid side chains and carbonyls in multiphase dispersed systems results in a modified reaction rate of volatile formation. ${ }^{12}$ The most relevant example is represented by the study of the time course of Maillard volatile products in binary structured fluids composed of monoacylglycerols and water, forming either water-in-oil (W/O), $\mathrm{O} / \mathrm{W}$ microemulsions, or bicontinuous systems. In such media, the combination of water activity at the surface of lipid droplets and the presence of surfactants lead to the formation of unique aroma compounds that, in aqueous environment, are not formed. ${ }^{13-15}$ In a similar

Received: March 2, 2020

Revised: April 17, 2020

Accepted: April 19, 2020

Published: April 19, 2020

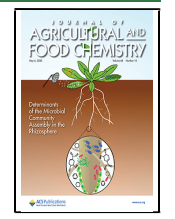


Table 1. Analytical Performances of the Compounds Identified in This Study ${ }^{a}$

\begin{tabular}{|c|c|c|c|c|c|c|c|}
\hline compound & chromatography & RT & $\mathrm{EC}$ & $\mathrm{TM}$ & EM & $\Delta(\mathrm{ppm})$ & $\lambda_{\max }$ \\
\hline alanine & HILIC $[\mathrm{M}+\mathrm{H}]^{+}$ & 4.5 & $\mathrm{C}_{3} \mathrm{H}_{7} \mathrm{NO}_{2}$ & 90.05496 & 90.05505 & 1.0 & \\
\hline Fru-Ala & HILIC $[\mathrm{M}+\mathrm{H}]^{+}$ & 4.1 & $\mathrm{C}_{9} \mathrm{H}_{17} \mathrm{NO}_{7}$ & 252.10778 & 252.10755 & -0.9 & \\
\hline pyridinium ion 1 & $\mathrm{RP}[\mathrm{M}]^{+}$ & 7.8 & $\mathrm{C}_{9} \mathrm{H}_{12} \mathrm{NO}_{4}^{+}$ & 198.07608 & 198.07618 & 0.5 & 298 \\
\hline pyridinium ion 2 & $\mathrm{RP}[\mathrm{M}]^{+}$ & 5.8 & $\mathrm{C}_{8} \mathrm{H}_{10} \mathrm{NO}_{3}^{+}$ & 168.06552 & 168.06556 & 0.2 & 285 \\
\hline
\end{tabular}

${ }^{a}$ Alanine and Fru-Ala were separated by HILIC, while pyridinium betaines were separated by C18 reversed-phase chromatography. Error (ppm) was calculated as the ratio between the difference of the theoretical mass (TM) minus the experimental mass (EM) and the theoretical mass, multiplied per one million. RT, retention time; $\lambda_{\max }$ UV absorbance; EC, elemental composition; alapyridaine, pyridinium ion 1; and 1-(1carboxyethyl)-3-hydroxy-pyridinium ion, pyridinium ion 2.

way, the use of amphiphilic lecithin and monoglycerides provides new functionalities through the formation of lipid selfassembled structures. These structures influence oxidation and Maillard-type reactions by increasing the yield of aroma compounds, as norfuraneol, when compared to the reaction performed in aqueous solutions. In particular, the addition of oil to a reversed bicontinuous structure formed by monoglycerides leads to a reversed microemulsion, which, considering its low viscosity, is particularly suitable for food products and acts as a reactor system. ${ }^{16}$

The use of emulsified systems can be instrumental to improve food naturalness, a crucial parameter for consumer choices. The technology and ingredients used in food processing constitute one of the three categories for defining "food naturalness". ${ }^{17}$ Among natural ingredients, amino acids and reducing sugars promote the formation of taste enhancers, which are able to increase the human sensitivity for sweetness, saltiness, and umami taste by modulating the perception of the basic taste modalities. ${ }^{18} N$-(1-carboxyethyl)-6(hydroxymethyl)pyridinium-3-ol inner salt (alapyridaine) is generated from alanine and glucose, and its $(+)-(S)$-isomer has been reported as the first taste enhancing product of the MR in beef broth. ${ }^{19}$ Along with intermediates such as Amadori compounds and volatile molecules, emulsions can be a suitable environment for the formation of nonvolatile taste enhancers. In this work, we explored the possibility to improve the formation of two pyridinium compounds starting from glucose and alanine through the combination of a surfactant-stabilized dispersed oil phase, thermal treatment, and an alkaline $\mathrm{pH}$. We introduced the possibility that the alkyl side chain of alanine can be used to boost the formation of taste-active molecules at the surface of oil droplets as a consequence of a fast degradation of glucose and the Amadori intermediate.

\section{MATERIALS AND METHODS}

Chemicals and Reagents. Polyoxyethylene (20) sorbitan monolaurate (Tween 20), sodium hydroxide, sodium dihydrogen phosphate, formic acid, tricaprylin, 1-(carboxymethyl)-5-hydroxy-2(hydroxymethyl)-pyridinium inner salt, L-alanine, and D-glucose were obtained from Sigma-Aldrich (St. Louis, MO); acetonitrile, methanol, and liquid chromatography mass spectrometry (LC-MS) grade water were obtained from Merck (Darmstadt, Germany). N-(1-deoxy-Dfructos-1-yl)-L-alanine (Fru-Ala) was obtained from Toronto Research Chemicals (North York, ON).

Reaction Model System for Identification of Pyridinium Compounds. Glucose was reacted with alanine by using 125 and 45 $\mathrm{mM}$, respectively, according to the procedure described by Wakamatsu and colleagues for $S$-allyl-cysteine and glucose. ${ }^{20}$ A liquid reaction mixture and a dried reaction mixture were preliminary prepared to characterize the main end products of the glucose and alanine reaction. In the liquid reaction system, alanine and glucose were dissolved into $62.5 \mathrm{mM}$ sodium dihydrogen phosphate $\mathrm{pH} 7.0$ adjusted with sodium hydroxide $1 \mathrm{M}$ (final volume, $5 \mathrm{~mL}$ ). For the dried reaction system, the liquid mixture was freeze-dried before thermal treatment. Samples were heated in an air forced circulating oven (Memmert, Schwabach, Germany) for $60 \mathrm{~min}$ at $180{ }^{\circ} \mathrm{C}$ and then cooled down. For both reaction mixtures, $2.5 \mathrm{~mL}$ of $80: 20$ water/methanol (v/v) was added to both liquid and dry cake samples, and then each was vortexed for $30 \mathrm{~s}$. The mixtures were filtered using $0.45 \mu \mathrm{m}$ PTFE filters (Phenomenex, Torrance, CA) and, for the dry method, diluted 1:5 with an additional water/methanol solution (80:20, v/v). Upon dilution, $10 \mu \mathrm{L}$ of each sample was injected onto the LC diode array detector (DAD) then onto high-resolution mass spectrometer (HRMS) system for analyses. For LC-DAD detection, samples were analyzed through an LC-10AD binary system (Shimadzu, Kyoto, Japan) equipped with an SPD-M10A diode array detector, SCL-10A controller (both from Shimadzu), and Series 200 autosampler (PerkinElmer, Carlsbad, CA). Chromatographic separation was achieved through a Synergi Fusion column $(150 \times 2.1$ $\mathrm{mm}, 4 \mu \mathrm{m}$, Phenomenex) by using mobile phases: $0.1 \%$ formic acid in water (A) and $0.1 \%$ formic acid in methanol (B). The following gradients $(\mathrm{min} / \% \mathrm{~B}):(0 / 5),(2.5 / 5),(5 / 40),(12 / 95)$, and $(15 / 95)$ were used, while the total flow was $0.3 \mathrm{~mL} / \mathrm{min}$. The same chromatographic conditions were implemented on an Exactive Orbitrap HRMS (Thermo Fisher, Bremen, Germany) to match UV spectra to mass spectrometry profiles; the mass spectrometry procedure is detailed in the section "Pyridinium Compound Quantitation".

Preparation of Model Systems. An unbuffered model system (MSM1) was made by solubilizing D-glucose monohydrate $(25 \mathrm{mM})$ and L-alanine $(4.5 \mathrm{mM})$ in water. The solution was stirred for $30 \mathrm{~min}$ at room temperature $(1000 \mathrm{rpm})$ to fully dissolve the two reagents. An aliquot of unbuffered MSM1 was used to prepare MSM2, and $1 \%$ of Tween $20(\mathrm{w} / \mathrm{w})$ and $10 \%(\mathrm{w} / \mathrm{w})$ of tricaprylin were added. In parallel, aqueous MSM3 and emulsified MSM4 were prepared by following the procedure described above but substituting water with alkaline buffer consisting of sodium dihydrogen phosphate $(62.5 \mathrm{mM})$ adjusted with sodium hydroxide $(1 \mathrm{M})$ at $\mathrm{pH}$ 9.5. For the oilcontaining systems (MSM2 and MSM4), emulsification was achieved in two steps. First, a coarse emulsion was prepared by using a T-18 ULTRA-TURRAX high-shear mixer (IKA, Staufen im Breisgau, Germany) at $11,000 \mathrm{rpm}$ for $40 \mathrm{~s}$. Second, the obtained coarse emulsion was passed through a high-pressure homogenizer (Microfluidizer; Microfluidics, Westwood, MA) at 800 bar for three times. The aqueous solutions MSM1 and MSM3 were also passed through the high-pressure homogenizer under the same conditions.

Thermal Treatments. An aliquot $(5 \mathrm{~mL})$ of each of the four model systems was transferred into a screw-capped flask $(13 \times 100,9$ $\mathrm{mL}$ approximative volume, DURAN, Mainz, Germany). Samples were heated in a heating block (Liebisch, Bielefeld, Germany) for 2, 4, 6, and $8 \mathrm{~min}$ at $140{ }^{\circ} \mathrm{C}$. After each cycle of thermal treatment, the heating block was equilibrated for $10 \mathrm{~min}$ at $140{ }^{\circ} \mathrm{C}$ before placing the subsequent series of samples. Samples were cooled down on ice immediately after heating $(10 \mathrm{~min})$ and, after physicochemical characterization, stored at $-20{ }^{\circ} \mathrm{C}$ until LC analyses.

Physical Characterization of the Emulsion Systems. Physical characterization was performed on the emulsion systems MSM2 and MSM4 at room temperature after thermal treatment and incubation on ice to stop the reaction. Mean droplet diameter, span, specific surface area, and droplet size distribution were determined by static 

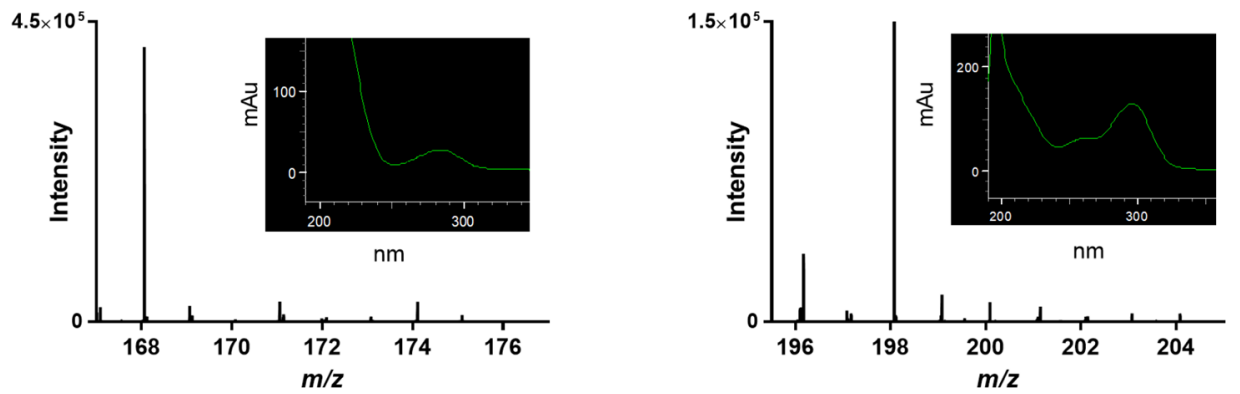

Figure 1. High-resolution mass spectra and UV absorbance spectra associated with the two pyridinium betaines. Left panel: 1-(1-carboxyethyl)-3hydroxy-pyridinium ion; right panel: alapyridaine.

light scattering (Mastersizer 3000; Malvern Instruments, Malvern, UK). Values used for the refractive indices were 1.33 for the continuous aqueous phase and 1.46 for tricaprylin. The samples were diluted in water to obtain an obscuration between 4 and $7 \%$.

Pyridinium Compound Quantitation. Target pyridinium betaine compounds were quantitated by LC-HRMS through a heated electrospray interface (HESI-II) operating in the positive ion mode. The current ion of the analytes listed in Table 1 was detected through reversed-phase chromatography according to the analytical setup for LC-DAD analysis used for alanine and glucose reaction mixture characterization described above. Ions were scanned in the $m / z$ range of 50-350, and the resolving power was set to 75,000 full width at half-maximum (FWHM, $m / z 200$ ), resulting in a scan time of $1 \mathrm{~s}$. The interface parameters were spray voltage $(4.2 \mathrm{kV})$, capillary voltage $(20.0 \mathrm{~V})$, capillary temperature $\left(300{ }^{\circ} \mathrm{C}\right)$, heater temperature $(300$ ${ }^{\circ} \mathrm{C}$ ), sheath gas flow (50 arbitrary units), and auxiliary gas flow (20 arbitrary units). This analytical setup was used for the detection of pyridinium compounds in emulsified, aqueous model systems and mass confirmation of reaction mixtures prepared for the identification of pyridinium betaines. Before filtering each sample in the presence of Tween 20, tricaprylin was diluted in methanol/water $(20: 80, \mathrm{v} / \mathrm{v})$ and then centrifuged $\left(21,000 \times g, 10 \mathrm{~min}, 4{ }^{\circ} \mathrm{C}\right)$, and the creamy phase was discarded. Quantitation of pyridinium betaines was performed by external calibration curves using 1-(carboxymethyl)-5-hydroxy-2(hydroxymethyl)-pyridinium inner salt as a reference standard.

Quantitation of Alanine and Fru-Ala. Alanine and Fru-Ala were detected by HILIC-HRMS following the procedure reported by Troise et al. with minor modifications. ${ }^{21}$ Samples $(0.1 \mathrm{~mL})$ were diluted with $4 \mathrm{~mL}$ of a ternary mixture of acetonitrile/water/formic acid $(85: 14.9: 0.1, \mathrm{v} / \mathrm{v} / \mathrm{v})$, and after centrifugation (at $4{ }^{\circ} \mathrm{C}$, for $15 \mathrm{~min}$ at $21,100 \times g)$, the creamy phase was discarded, and the samples were further diluted and filtered by using RC cellulose filters $(0.22 \mu \mathrm{m}$, Phenomenex). The chromatographic separation of alanine and FruAla was achieved by three mobile phases consisting of $0.05 \%$ formic acid in acetonitrile (solvent A), $0.05 \%$ formic acid in water (solvent $\mathrm{B}$ ), and $50 \mathrm{mM}$ ammonium formate (solvent $\mathrm{C}$ ). The following linear gradients of solvent B ( $\mathrm{min} / \% \mathrm{~B}):(0 / 2),(1.20 / 2),(4 / 40)$, and (6/ $40)$ were used, while the mobile phase $C$ was constant at $10 \%$. The flow rate was set to $0.4 \mathrm{~mL} / \mathrm{min}$, and the injection volume was $5 \mu \mathrm{L}$. A thermostated $\left(35^{\circ} \mathrm{C}\right)$ Kinetex $2.6 \mu \mathrm{m}(75 \times 2.1 \mathrm{~mm})$ core-shell silica HILIC column and UHPLC HILIC guard column (Phenomenex) were used to guarantee the optimal separation between the intermediate (Fru-Ala) and its two precursors (glucose and alanine). The Accela 1250 UHPLC system (Thermo Fisher Scientific) was directly interfaced to an Exactive Orbitrap HRMS (Thermo Fisher Scientific), and target analytes were detected through a heated electrospray interface (HESI-II, positive mode) in the $\mathrm{m} / \mathrm{z}$ range of 60-400. Resolving power, injection time, and scan time were set according to previous conditions for pyridinium identifications. The interface parameters were spray voltage $(4.5 \mathrm{kV})$, capillary voltage $(20.5 \mathrm{~V})$, skimmer voltage $(16 \mathrm{~V})$, tube lens voltage $(110 \mathrm{~V})$, capillary temperature $\left(295{ }^{\circ} \mathrm{C}\right)$, heater temperature $\left(300{ }^{\circ} \mathrm{C}\right)$, sheath gas flow (42 arbitrary units), and auxiliary gas flow (6 arbitrary units). The calibration procedure and analytical performances were monitored according to Troise et al. ${ }^{21}$
Statistical Analysis. Target analytes were monitored in four independent $\mathrm{O} / \mathrm{W}$ emulsions and control systems (buffered and unbuffered) upon thermal treatment. The results were expressed as $\mathrm{mmol} / \mathrm{L}$ for alanine or $\mu \mathrm{mol} / \mathrm{L}$ for Fru-Ala and pyridinium betaines. The time course profile, Tukey test for statistical analysis $(\alpha=0.05)$, and paired $t$ test for molar yield of pyridinium betaines were monitored using GraphPad (Prism, La Jolla, CA). The results of the Tukey test are summarized in Table S1.

\section{RESULTS AND DISCUSSION}

Identification of Pyridinium Betaine Derivatives. Figure 1 shows the mass and UV absorbance spectra of the two target pyridinium betaines upon the reaction between alanine and glucose, freeze-dried and thermally treated at 180 ${ }^{\circ} \mathrm{C}$ for $60 \mathrm{~min}$. This procedure was used as the reference for the tentative identification of MR products, 1-(1-carboxyethyl)-5-hydroxy-2-(hydroxymethyl)-pyridinium ion (alapyridaine) and 1-(1-carboxyethyl)-3-hydroxy-pyridinium ion; the same chromatographic and mass spectrometry profiles were obtained during thermal treatments at $140{ }^{\circ} \mathrm{C}$ up to $8 \mathrm{~min}$ with emulsion and aqueous model systems. Alapyridaine was characterized by a maximum absorbance at $298 \mathrm{~nm}$, while 1(1-carboxyethyl)-3-hydroxy-pyridinium ion had a maximum absorbance at slightly lower wavelength. The retention time and chromatographic separation were consistent with the use of reversed-phase chromatography (5.6 and $7.8 \mathrm{~min}$, respectively). HRMS trials revealed for both compounds an exact mass $[\mathrm{M}]^{+}$up to the fifth decimal digit of 198.07618 and 168.06556, respectively, with an error lower than $0.6 \mathrm{ppm}$ in both cases (Table 1). The experimental isotopic distribution was in line with the theoretical isotopic distribution, supporting the hypothesis that both compounds were pyridinium betaine derivatives arisen from the reaction of alanine and glucose or one of its fragmentation products. Mass spectrometry information and UV spectra were in accordance with the study of Soldo and co-workers who synthesized and purified 16 pyridinium betaines. ${ }^{19}$ Regarding alapyridaine, the results here reported matched those obtained by Ottinger and co-workers for the structure determination of sweet taste enhancer: at an acidic $\mathrm{pH}$ in the presence of formic acid in the mobile phase, alapyridaine exhibited the typical UV spectrum with only a sole maximum absorbance at $\lambda=298 \mathrm{~nm} .{ }^{22}$

Physicochemical Characterization of Emulsion Systems. We investigated two model emulsions with tricaprylin and Tween 20 (a nonionic surfactant), one unbuffered (MSM2, final pH 6.5) and another one with sodium phosphate buffer at $\mathrm{pH} 9.5$ (MSM4) to gain insights into the formation of alanine derivatives in the presence of a large oil-water interfacial area. Both model systems were compared to their respective controls without dispersed lipids and without 
0 min

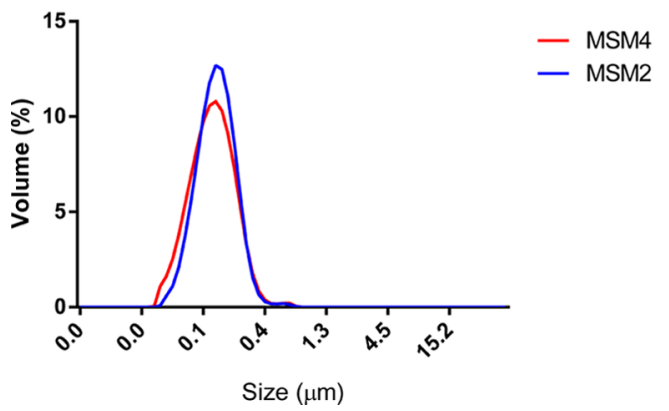

$8 \mathrm{~min}$

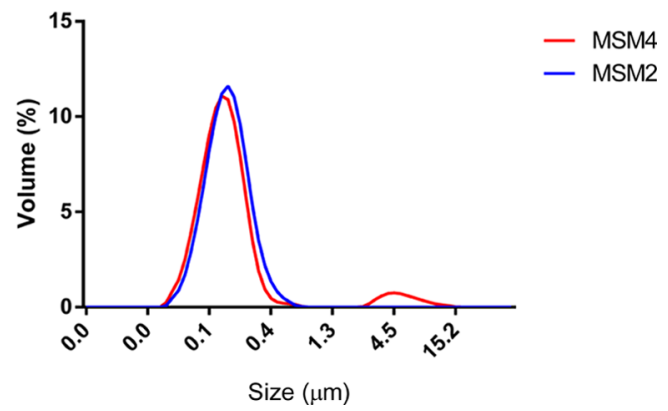

Figure 2. Particle size distribution of emulsions: MSM2 (unbuffered emulsified system, $\mathrm{pH}$ 6.5) and MSM4 (emulsified model system, $\mathrm{pH}$ 9.5) before the thermal treatment (freshly prepared emulsions, $0 \mathrm{~min}$ ) and after $8 \mathrm{~min}$ at $140{ }^{\circ} \mathrm{C}$.
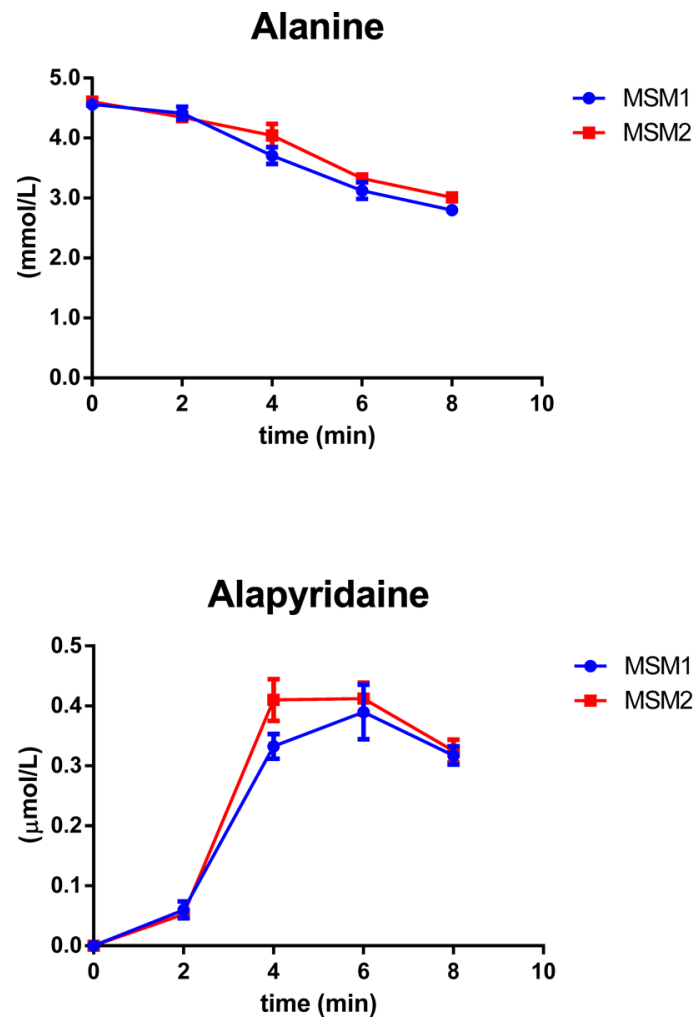

\section{Fru-Ala}

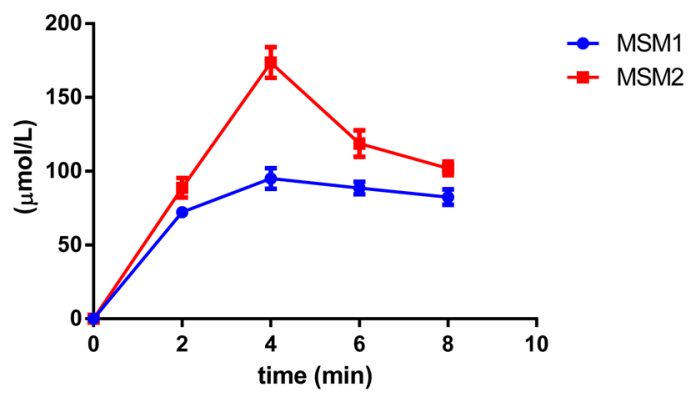

\section{1-(1-carboxyethyl)-3-hydroxy-pyridinium ion}

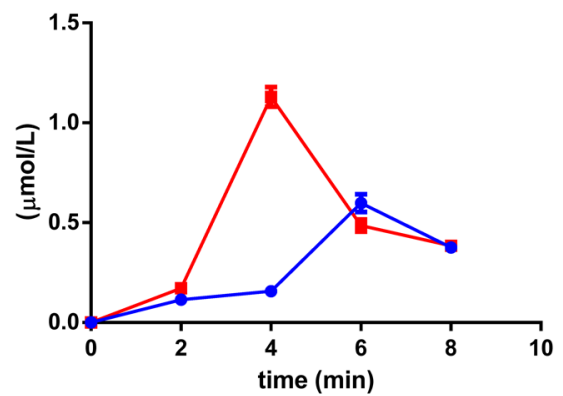

Figure 3. Time course profile of alanine, Fru-Ala, 1-(1-carboxyethyl)-5-hydroxy-2-(hydroxymethyl)-pyridinium ion (alapyridaine), and 1-(1carboxyethyl)-3-hydroxy-pyridinium ion (pyridinium ion); both systems were prepared in an unbuffered aqueous continuous phase with a final $\mathrm{pH}$ of 6.5. Red squares correspond to the emulsion system (Tween 20-stabilized tricaprylin droplets, MSM2), while blue dots correspond to the aqueous unbuffered solution (MSM1). Significant statistical differences (two-way ANOVA, Tukey test, $\alpha<0.05$ ) are reported in Table S1.

Tween, and each of them was thermally treated at $140{ }^{\circ} \mathrm{C}$ up to $8 \mathrm{~min}$. Figure 2 shows the droplet size distribution in $\mathrm{O} / \mathrm{W}$ emulsions at $\mathrm{pH} 6.5$ (unbuffered, MSM2) and at $\mathrm{pH} 9.5$ (MSM4). The left panel reports the droplet size distribution in both model systems freshly homogenized before thermal treatment $(0 \mathrm{~min})$, whereas the right panel shows the droplet size distribution of the same emulsions after $8 \mathrm{~min}$ at $140{ }^{\circ} \mathrm{C}$. For MSM2 (unbuffered emulsion), the population of droplets was monomodal, and this did not change upon thermal treatment. At 0 and $8 \mathrm{~min}$, both size distributions were centered approximately at a $0.15 \mu \mathrm{m}$ diameter, in line with our previous paper. ${ }^{6}$ On the opposite, while the alkaline emulsion (MSM4) initially had a droplet size distribution similar to that of the unbuffered emulsion, the distribution became bimodal upon thermal treatment: a shoulder appeared at an approximately $4.5 \mu \mathrm{m}$ diameter, amounting for $0.6 \%$ of total volume. It should still be pointed out that no excessive emulsion destabilization occurred as the initial peak corresponding to small droplets was still largely dominant. The use of a nonionic surfactant was probably a good choice herein as the physical stability of protein- or ionizable surfactantstabilized emulsions can be largely compromised by heat treatments and $\mathrm{pH}$ changes, ${ }^{23}$ while in our model systems with a nonionic surfactant, the formation of larger population can be mainly attributed to the high temperature used. ${ }^{24}$

Maillard Reaction in Slightly Acidic or Alkaline Environment. Figure 3 highlights the degradation of alanine, formation and degradation of Fru-Ala, and profile of alapyridaine and 1-(1-carboxyethyl)-3-hydroxy-pyridinium ion formation in MSM1 and MSM2. The initial concentration of 
Alanine
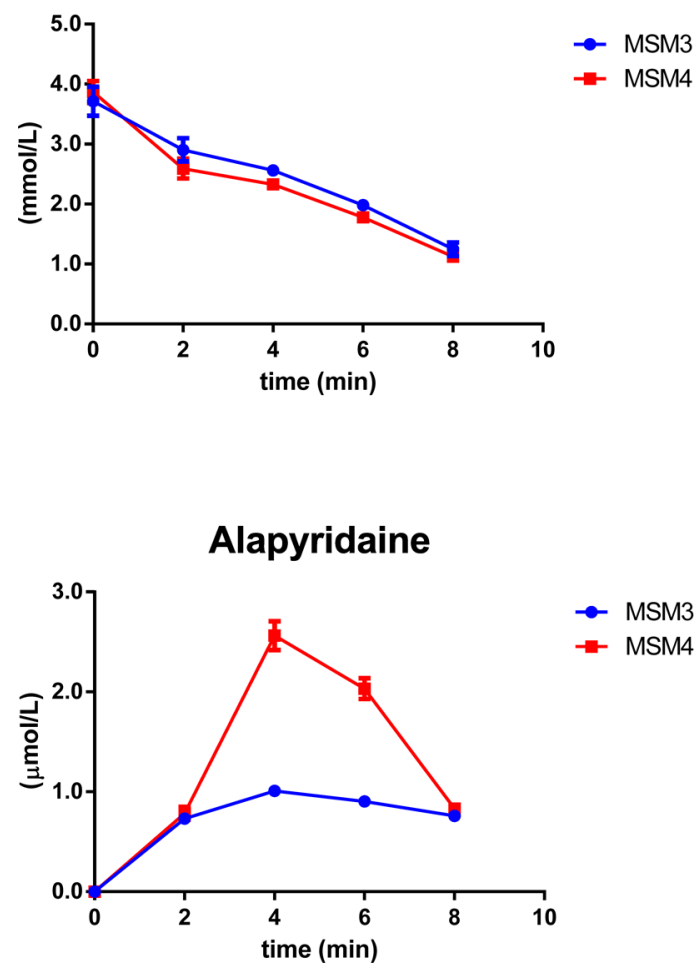

Fru-Ala

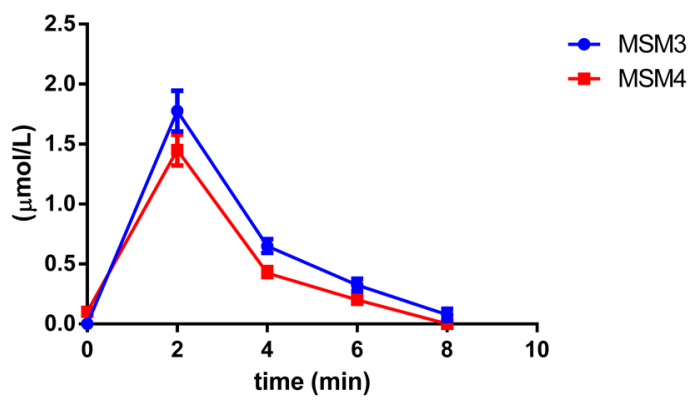

1-(1-carboxyethyl)-3-hydroxy-pyridinium ion

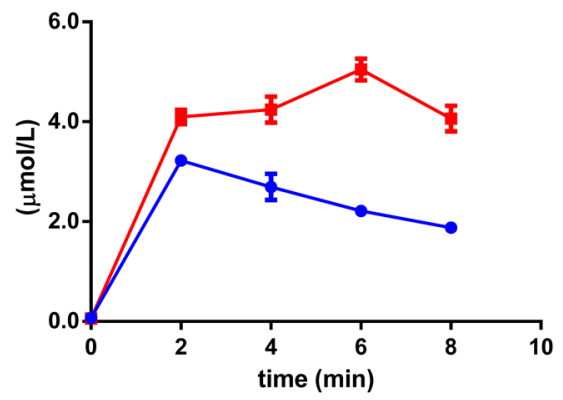

Figure 4. Time course profile of the four analytes monitored: alanine, Fru-Ala, 1-(1-carboxyethyl)-5-hydroxy-2-(hydroxymethyl)-pyridinium ion (alapyridaine), and 1-(1-carboxyethyl)-3-hydroxy-pyridinium ion; both systems were prepared at alkaline $\mathrm{pH}$ (9.5). Red squares correspond to the emulsion system (Tween 20-stabilized tricaprylin droplets, MSM4), while blue dots correspond to the aqueous unbuffered solution (MSM3). Significant statistical differences (two-way ANOVA, Tukey test, $\alpha<0.05$ ) are reported in Table S1.

alanine was $4.56 \pm 0.03$ and $4.61 \pm 0.08 \mathrm{mmol} / \mathrm{L}$ for aqueous and emulsion systems, respectively. In both cases, at the end of thermal treatment, the final concentration dropped down to $2.80 \pm 0.08$ and $3.01 \pm 0.08 \mathrm{mmol} / \mathrm{L}$, respectively, thus with a reduction close to $40 \%$. When comparing MSM1 and MSM2, significant differences in alanine degradation were observed only at $4 \mathrm{~min}$ (Table S1). However, the presence of Tween 20stabilized tricaprylin droplets significantly influenced the formation of the Amadori compound already after $2 \mathrm{~min}$; indeed at $4 \mathrm{~min}$ in the aqueous environment, the concentration was $95.12 \pm 6.97 \mu \mathrm{mol} / \mathrm{L}$, while in the presence of emulsions, it jumped to $173.61 \pm 10.37 \mu \mathrm{mol} / \mathrm{L}$, thus an $83 \%$ increase. At $6 \mathrm{~min}$, the concentration in both model systems decreased, suggesting that Fru-Ala was converted in its end products, and even if after $8 \mathrm{~min}$, the concentration in emulsions was still $24 \%$ higher than the aqueous model system, implying higher yield in the presence of oil droplets. A similar trend was observed for alapyridaine: the highest concentration was obtained in the emulsion system after $4 \mathrm{~min}(0.41 \pm 0.03$ $\mu \mathrm{mol} / \mathrm{L})$, while at 6 and $8 \mathrm{~min}$, no significant difference was present between both model systems. In the presence of emulsified tricaprylin, the concentration of 1-(1-carboxyethyl)3-hydroxy-pyridinium ion was 6.1 times higher than in aqueous systems after $4 \mathrm{~min}$, and then its concentration suddenly decreased, reaching a concentration of $0.38 \pm 0.02 \mu \mathrm{mol} / \mathrm{L}$ at the end of the thermal treatment, highlighting the possibility of further decomposition or association with pyridinium betaines.

Figure 4 reports the time course profile of the four investigated compounds in alkaline conditions, i.e., in MSM3 (aqueous environment) and MSM4 (emulsion). For these experiments, alanine and glucose solutions were prepared in the same conditions as for the unbuffered model system, except the presence of sodium dihydrogen phosphate and sodium hydroxide. The concentration of alanine in MSM3 and MSM4 at the beginning of the thermal treatment was $20 \%$ lower than in MSM1 and MSM2, meaning that during the high-pressure homogenization at alkaline $\mathrm{pH}$, the increase in temperature promoted further reactions on the alanine moiety. Substantial differences were observed for alanine degradation during thermal treatment in the presence or absence of oil droplets at alkaline $\mathrm{pH}$ : in MSM3 and MSM4, alanine concentration was significantly different at 2 and $4 \mathrm{~min}$. Alanine concentration in MSM3 over the thermal treatment ranged from $3.71 \pm 0.24 \mathrm{mmol} / \mathrm{L}$ at the beginning down to $1.25 \pm 0.11 \mathrm{mmol} / \mathrm{L}$ after $8 \mathrm{~min}$, while in MSM4, it ranged from $3.87 \pm 0.18 \mathrm{mmol} / \mathrm{L}$ down to $1.12 \pm 0.09 \mathrm{mmol} / \mathrm{L}$. The oil influenced the formation of Fru-Ala as well: at the beginning of the process, the Amadori compound concentration was $0.04 \mu \mathrm{mol} / \mathrm{L}$ in the aqueous system (MSM3), while in the emulsified alkaline environment (MSM4), it was $69 \%$ higher. The $\mathrm{pH}$ strongly favored the degradation of FruAla: its concentration sharply decreased already after $2 \mathrm{~min}$ in both MSM3 and MSM4 and was largely lower than with the unbuffered systems. After $2 \mathrm{~min}$, the alapyridaine concentration doubled the highest concentration observed in the unbuffered systems. In the presence of oil droplets, at $4 \mathrm{~min}$, the alapyridaine concentration was $2.56 \pm 0.14 \mu \mathrm{mol} / \mathrm{L}$, i.e., 2.5 times higher than in its alkaline control sample without oil droplets. These differences decreased after $4 \mathrm{~min}$, and upon 8 min at $140{ }^{\circ} \mathrm{C}$, the difference was only $10 \%$. The presence of oil droplets significantly impacted the formation of 1-(1carboxyethyl)-3-hydroxy-pyridinium ion throughout the ther- 

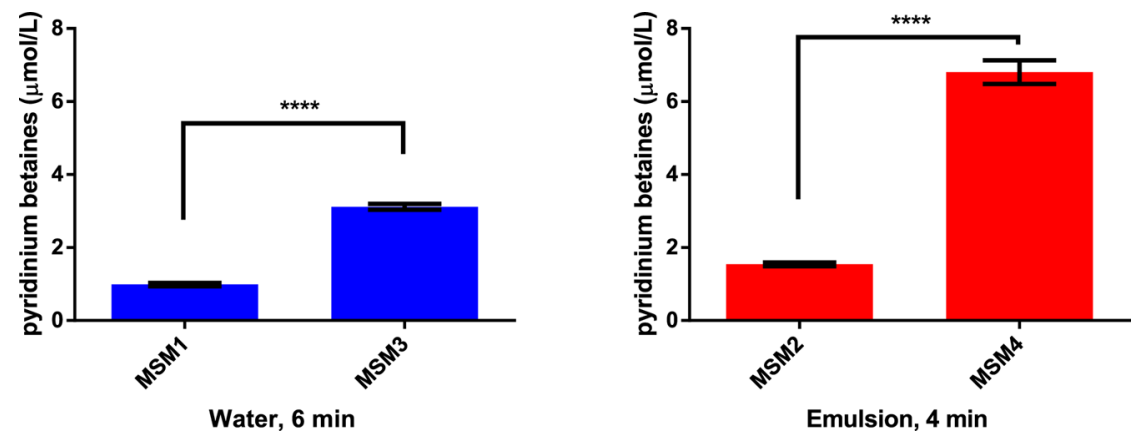

Figure 5. Sum of both pyridinium betaine compounds formed: (red) in emulsions after 4 min or (blue) in aqueous solution after 6 min. Both reaction times were selected to compare the highest concentration of alapyridaine in unbuffered solution (6 min) or in unbuffered emulsion (4 min). Significant differences $(p$ value $<0.0001)$ were monitored through a paired $t$ test.

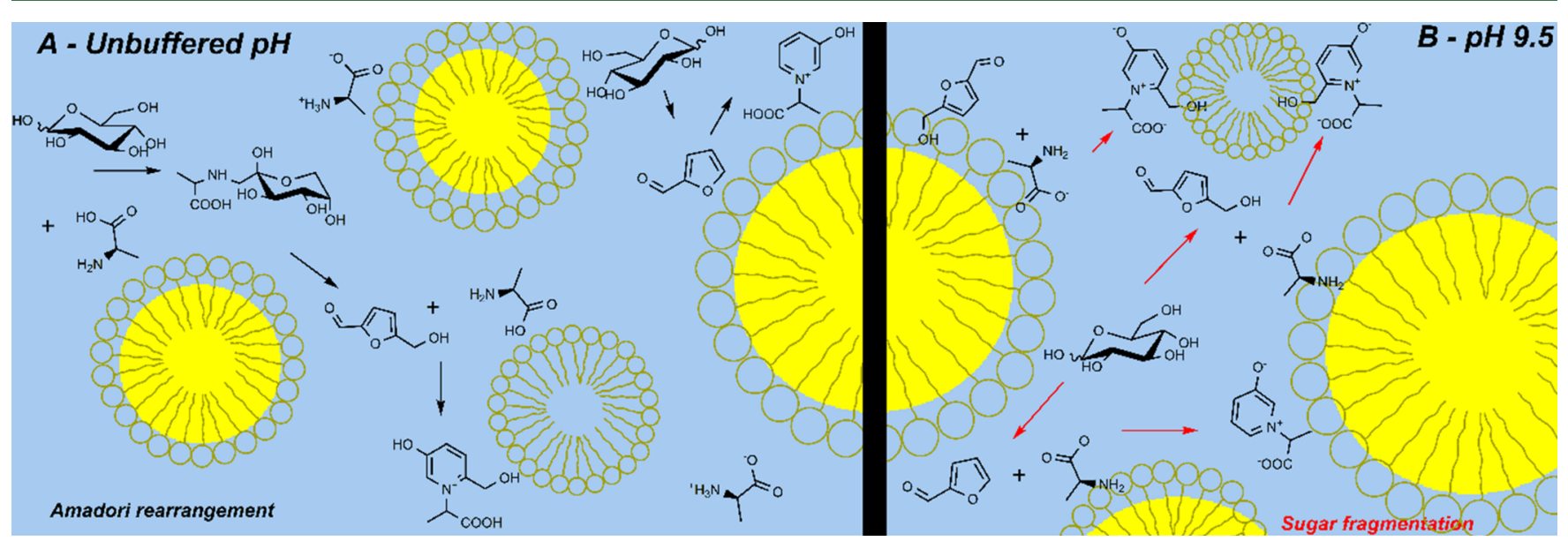

Figure 6. Proposed reaction mechanisms of pyridinium betaine formation with emphasis on reactants' location at the $\mathrm{O} / \mathrm{W}$ interface. In pale yellow, Tween 20-covered interface; yellow, tricaprylin droplets; red arrows highlight faster degradation. (A) unbuffered model system. (B) Alkaline $\mathrm{pH}$. In both environments, the putative presence of surfactant micelles is outlined.

mal treatment: compared to the alkaline aqueous system, and the increase ranged from $27 \%$ after 2 min up to $128 \%$ higher at $6 \mathrm{~min}$. Although for the alkaline aqueous system, the decrease in pyridinium ion concentration started after $2 \mathrm{~min}$, and in the case of alkaline emulsions, its concentration reached a maximum after $6 \mathrm{~min}(5.04 \pm 0.21 \mu \mathrm{mol} / \mathrm{L})$ then slightly decreased with a final concentration of $4.06 \pm 0.26 \mu \mathrm{mol} / \mathrm{L}$. The combination of emulsions and alkaline $\mathrm{pH}$ promoted the formation of the two pyridinium betaines, as summarized in Figure 5. The sum of the two compounds at $4 \mathrm{~min}$ (highest concentration of the two compounds in the unbuffered system, MSM2) was compared to MSM4 at 4 min to better highlight the differences in the molar quantities formed. The combined concentration of both compounds was 4.4 times higher in MSM4 than in MSM2. The same layout was used for aqueous systems: the concentration of both betaines at $6 \mathrm{~min}$ in MSM3 was 3.1 times higher than in MSM1.

Chemical Significance of Emulsion Systems on the Formation of Pyridinium Betaines. Figure 6 summarizes the reaction pathways leading to the formation of pyridinium betaines in two different conditions, unbuffered (A) and alkaline $\mathrm{pH}$ (B, $\mathrm{pH}$ 9.5). Despite its negative $\log P(-0.574 \pm$ 0.272 , SciFinder, CAS), alanine is characterized by the presence of an alkyl moiety that can potentially interact with nonpolar oil droplets, while the amino and carboxylic groups can interact with the polar nonionic head of Tween 20. This would be consistent with the fact that in MSM2, the presence of lipid droplets accelerated the formation of Fru-Ala and promoted higher yields compared to the aqueous environment; these evidences are also in line with Maillard processes in binary structured fluids composed of monoacylglycerols and water, forming microemulsions. ${ }^{14}$ Moreover, the behavior of alanine is in line with that described for leucine in nonionic microemulsions: reactants were oriented at the interface, and water activity was adjusted using blends of surfactant and propylene glycol, resulting in unique aroma compound formation. $^{25}$ The use of alanine and tricaprylin also followed a previous study from our group, where phenylalanine and leucine were reacted with glucose in surfactant-stabilized emulsions, and their reactivity was modulated by their partition coefficient and specific location. We already reported that the combination of nonionic surfactants, saturated triglyceride oil droplets, and amino acids with hydrophobic side chains, such as leucine, led to modifications of the reactivity of Maillard precursors and to the formation of $\mathrm{N}$-(1-deoxy-D-fructos-1-yl)L-leucine controlled by promoting the location of amino acids and Amadori compounds at the lipid-water interface. Conversely, nonionic surfactants and saturated lipid droplets have only a minor impact on the formation of N-(1-deoxy-Dfructos-1-yl)-L-phenylalanine. ${ }^{6}$

It can be pointed out that the two targeted compounds were presumably formed, even in the presence of emulsions, according to the synthesis strategy previously described. ${ }^{22,26}$ Hydroxymethylfurfural (HMF) and 2-furaldehyde are the key 
intermediates that supervise the formation of both alapyridaine and 1-(1-carboxyethyl)-3-hydroxy-pyridinium ion, respectively. ${ }^{19}$ In particular for the latter, besides its ability to form oligomers, ${ }^{27}$ we hypothesized a possible direct contribution of surfactant/emulsions to the formation of 2-furaldehyde starting from the intact skeleton of glucose through the $\beta$-dicarbonyl route with 3-deoxyhexos-2-ulose as intermediates. ${ }^{28}$ In general, carbohydrate degradation is another active route that supports the release of both pyridinium ions. ${ }^{29,30}$

When an alkaline $\mathrm{pH}$ is used, the degradation of amino acids and reducing sugars follows specific routes, ${ }^{31}$ and in our case, alanine degradation was more pronounced in the alkaline medium than in unbuffered one. LC-HRMS full scan chromatograms in MSM2 and MSM4 after 4 min showed a degradation of polar compounds, poorly retained in reversedphase chromatography (such as glucose or alanine; details are shown in Figure $\mathrm{S} 1$ ). At alkaline $\mathrm{pH}$, in opposition to what was expected, Tween 20 bares saturated triglyceride, and it shows a negative charge, ${ }^{24,32,33}$ while glucose is essentially unprotonated, and its degradation mainly proceeds through Maillardinduced fragmentation and hydrolytic $\beta$-dicarbonyl cleavage. ${ }^{34}$ As a direct consequence of the charge state, the unprotonated form of the amino group of alanine (strongest basic $\mathrm{p} K_{\mathrm{a}}, 9.45$ ) combined to the open chain of the glucose is the major driver for the degradation of alanine, formation and degradation of Fru-Ala, and higher yields of pyridinium betaines as previously observed in glycine and glucose $\mathrm{e}^{35}$ or in sugar enolization at high $\mathrm{pH}$ in the presence of amino acids. ${ }^{36}$ Moreover, the alanine amino group can be poorly solvated at high $\mathrm{pH}$ when such a nucleophile is present in the polar head group of Tween 20 as it was already demonstrated for "naked anions" in alcohol ethoxylate microemulsions. ${ }^{37}$ The presence of emulsions switched the reaction routes to a faster degradation of FruAla: the same behavior was described by Yaghmur and coworkers for cysteine furfural reactions in self-assembled nanosized structured systems. ${ }^{38}$ In this respect, the definition of reaction routes for HMF and 2-furaldehyde as precursors of alapyridaine and 1-(1-carboxyethyl)-3-hydroxy-pyridinium ion becomes a relevant aspect. We hypothesized that Fru-Ala, but in particular sugar enolization and fragmentation, generated simultaneously HMF and 2-furaldehyde, ${ }^{39}$ and the presence of oil droplets accelerated both routes; in a closed system, aqueous reactants were forced to interact with each other or with amino residues at the interface as a consequence of the presence of emulsion droplets, hence resulting in a faster formation of the two fragmentation products, as previously observed for sucrose esters. ${ }^{13}$ A direct relationship between tricaprylin and end product formation cannot be excluded: the addition of glycerol to alanine and glucose or fructose is able to influence both physicochemical environments and to promote the inclusion of the glycerol moiety in volatile compounds with 2-oxopropanal as the intermediate. ${ }^{40}$

Alapyridaine and 1-(1-carboxyethyl)-3-hydroxy-pyridinium ion are both negatively charged at alkaline $\mathrm{pH}$, and as for their precursors, in the presence of negatively charged Tween 20covered tricaprylin droplets, we hypothesize that both are located within the aqueous layer, resulting in a possible enhancement of interaction with other molecules formed during the thermal treatment. Specifically, charged moieties, as in the case of pyridinium betaines, can form strong ion-dipole interactions with the surrounding water molecules, whereas the nonionized form can only form weaker dipole-dipole interactions: this situation results in a more pronounced partitioning of target taste-active molecules in the aqueous layer. Moreover, the role of Tween 20 can explain some of the results obtained: $1 \%$ of Tween present in the continuous phases can form micelles that may solubilize nonpolar molecules in their hydrophobic interior or amphiphilic molecules as inserted between the hydrophilic head groups. ${ }^{41}$ The result would be an increased affinity of nonpolar betaines for the aqueous phase, and this hypothesis will be tested in future studies. Taking into account that at $4 \mathrm{~min}$, the concentration of alapyridaine was perfectly in line with that obtained in beef broth ${ }^{42}$ but lower than that used for human perception of sweet, umami, and salty taste, ${ }^{18}$ it is worth mentioning that independently of the $\mathrm{pH}$ conditions, the concentration of the two pyridinium betaines decreased after 4 min, with the exception of 1-(1-carboxyethyl)-3-hydroxypyridinium ion in MSM4. A putative explanation arises from the high temperature used or to the strong alkaline conditions.

The relationship between the MR, glycation compounds, and nonvolatile taste-active molecules in emulsion systems is still underexplored, and several events can modulate the reaction rates and yields of target compounds. Compartmentalization and partitioning of reactants play a key role in the distribution of molecules between the oil phase, aqueous phase, and the $\mathrm{O} / \mathrm{W}$ interface. Here, we reported for the first time the effects of multiphase systems on the formation of nonvolatile, taste-active end products. We demonstrated that the reaction pathways leading to the formation of pyridinium betaines can be influenced by the combination of alkaline $\mathrm{pH}$ and saturated triglyceride droplets in the presence of a nonionic surfactant. We introduced a new approach for the study of the MR in dispersed environments in which the charge state of the precursors can be used to modulate the equilibrium between different regions and promote the interaction between amino acids and glucose or its degradation products. Although we did not study the sensory impact of betaine dynamics along with their formation in model systems, our findings support the possibility to develop technological strategies to boost sensory quality of foods in the actual context of clean-label demand by consumers. Moreover, our findings can be applied to develop new ingredients able to modulate the formation of taste-active compounds.

\section{ASSOCIATED CONTENT}

\section{SI Supporting Information}

The Supporting Information is available free of charge at https://pubs.acs.org/doi/10.1021/acs.jafc.0c01446.

Total ion current HRMS chromatogram in the positive mode in reversed-phase chromatography for the four model systems after $4 \mathrm{~min}$ and two-way ANOVA (Tukey test, $\alpha<0.05$ ) for the four model systems (PDF)

\section{AUTHOR INFORMATION}

\section{Corresponding Author}

Antonio Dario Troise - Department of Agricultural Sciences, University of Naples Federico II, Naples 80055, Italy; Proteomics \& Mass Spectrometry Laboratory, ISPAAM, National Research Council, Naples 80147, Italy; 이이.org/ 0000-0001-7635-5244; Phone: +39 081 5966006; Email: antoniodario.troise2@unina.it 


\section{Authors}

Claire C. Berton-Carabin - Food Process Engineering Group, Wageningen University, Wageningen 6708 WG, The Netherlands

Paola Vitaglione - Department of Agricultural Sciences, University of Naples Federico II, Naples 80055, Italy

Vincenzo Fogliano - Food Quality and Design Group, Wageningen University, Wageningen 6708 WG, The

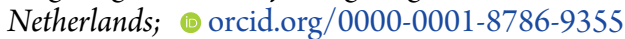

Complete contact information is available at: https://pubs.acs.org/10.1021/acs.jafc.0c01446

\section{Funding}

The research of A.D.T. was supported by University of Naples (UNINA, Italy) and Compagnia di San Paolo, in the frame of Program "STAR-L1" (Sostegno Territoriale alle Attivitá di Ricerca, Junior Principal Investigator Grants, University of Naples, Italy).

\section{Notes}

The authors declare no competing financial interest.

\section{ABBREVIATIONS}

MR, Maillard reaction; AP, Amadori product; Fru-Ala, N-(1deoxy-D-fructos-1-yl)-L-alanine; HRMS, high-resolution mass spectrometry; HILIC, hydrophilic interaction liquid chromatography

\section{REFERENCES}

(1) van Boekel, M.; Fogliano, V.; Pellegrini, N.; Stanton, C.; Scholz, G.; Lalliie, S.; Somoza, V.; Knorr, D.; Jasti, P. R.; Eisenbrand, G. A Review on the Beneficial Aspects of Food Processing. Mol. Nutr. Food Res. 2010, 54, 1215-1247.

(2) Hellwig, M.; Henle, T. Baking, Ageing, Diabetes: A Short History of the Maillard Reaction. Angew. Chem., Int. Ed. 2014, 53, 10316-10329.

(3) Capuano, E.; Oliviero, T.; van Boekel, M. A. J. S. Modeling Food Matrix Effects on Chemical Reactivity: Challenges and Perspectives. Crit. Rev. Food Sci. Nutr. 2018, 58, 2814-2828.

(4) Yaylayan, V. A. Classification of the Maillard Reaction: A Conceptual Approach. Trends Food Sci. Technol. 1997, 8, 13-18.

(5) Bel-Rhlid, R.; Berger, R. G.; Blank, I. Bio-Mediated Generation of Food Flavors - Towards Sustainable Flavor Production Inspired by Nature. Trends Food Sci. Technol. 2018, 78, 134-143.

(6) Troise, A. D.; Berton-Carabin, C. C.; Fogliano, V. Amadori Products Formation in Emulsified Systems. Food Chem. 2016, 199, 51-58.

(7) Berton-Carabin, C. C.; Schroën, K. Pickering Emulsions for Food Applications: Background, Trends, and Challenges. Annu. Rev. Food Sci. Technol. 2015, 6, 263-297.

(8) McClements, D. J.; Decker, E. A. Lipid Oxidation in Oil-inWater Emulsions: Impact of Molecular Environment on Chemical Reactions in Heterogeneous Food Systems. J. Food Sci. 2000, 65, $1270-1282$.

(9) Berton-Carabin, C. C.; Ropers, M. H.; Genot, C. Lipid Oxidation in Oil-in-Water Emulsions: Involvement of the Interfacial Layer. Compr. Rev. Food Sci. Food Saf. 2014, 13, 945-977.

(10) Decker, E. A.; McClements, D. J.; Bourlieu-Lacanal, C.; Durand, E.; Figueroa-Espinoza, M. C.; Lecomte, J.; Villeneuve, P. Hurdles in Predicting Antioxidant Efficacy in Oil-in-Water Emulsions. Trends Food Sci.Technol. 2017, 67, 183-194.

(11) Sagalowicz, L.; Michel, M.; Blank, I.; Schafer, O.; Leser, M. E. Self-Assembly in Food - A Concept for Structure Formation Inspired by Nature. Curr. Opin. Colloid Interface Sci. 2017, 28, 87-95. (12) Garti, N. Microemulsions as Microreactors for Food Applications. Curr. Opin. Colloid Interface Sci. 2003, 8, 197-211.
(13) Fanun, M.; Leser, M.; Aserin, A.; Garti, N. Sucrose Ester Microemulsions as Microreactors for Model Maillard Reaction. Colloids Surf., A 2001, 194, 175-187.

(14) Vauthey, S.; Milo, C.; Frossard, P.; Garti, N.; Leser, M. E.; Watzke, H. J. Structured Fluids as Microreactors for Flavor Formation by the Maillard Reaction. J. Agric. Food Chem. 2000, 48, 4808-4816.

(15) Newton, A. E.; Fairbanks, A. J.; Golding, M.; Andrewes, P.; Gerrard, J. A. The Influence of Emulsion Structure on the Maillard Reaction of Ghee. Food Chem. 2015, 173, 1243-1249.

(16) Sagalowicz, L.; Moccand, C.; Davidek, T.; Ghanbari, R.; Martiel, I.; Negrini, R.; Mezzenga, R.; Leser, M. E.; Blank, I.; Michel, M. Lipid Self-Assembled Structures for Reactivity Control in Food. Philos. Trans. R. Soc., A 2016, 374, 20150136.

(17) Román, S.; Sánchez-Siles, L. M.; Siegrist, M. The Importance of Food Naturalness for Consumers: Results of a Systematic Review. Trends Food Sci. Technol. 2017, 67, 44-57.

(18) Hofmann, T. Taste-Active Maillard Reaction Products: The "Tasty" World of Nonvolatile Maillard Reaction Products. Ann. N. Y. Acad. Sci. 2005, 1043, 20-29.

(19) Soldo, T.; Frank, O.; Ottinger, H.; Hofmann, T. Systematic Studies of Structure and Physiological Activity of Alapyridaine. A Novel Food-Born Taste Enhancer. Mol. Nutr. Food Res. 2004, 48, 270-281.

(20) Wakamatsu, J.; Stark, T. D.; Hofmann, T. Taste-Active Maillard Reaction Products in Roasted Garlic (Allium Sativum). J. Agric. Food Chem. 2016, 64, 5845-5854.

(21) Troise, A. D.; Wiltafsky, M.; Fogliano, V.; Vitaglione, P. The Quantification of Free Amadori Compounds and Amino Acids Allows to Model the Bound Maillard Reaction Products Formation in Soybean Products. Food Chem. 2018, 247, 29-38.

(22) Ottinger, H.; Soldo, T.; Hofmann, T. Discovery and Structure Determination of a Novel Maillard-Derived Sweetness Enhancer by Application of the Comparative Taste Dilution Analysis (CTDA). J. Agric. Food Chem. 2003, 51, 1035-1041.

(23) McClements, D. J. Protein-Stabilized Emulsions. Curr. Opin. Colloid Interface Sci. 2004, 9, 305-313.

(24) Teo, A.; Goh, K. K. T.; Wen, J.; Oey, I.; Ko, S.; Kwak, H. S.; Lee, S. J. Physicochemical Properties of Whey Protein, Lactoferrin and Tween 20 Stabilised Nanoemulsions: Effect of Temperature, PH and Salt. Food Chem. 2016, 197, 297-306.

(25) Lutz, R.; Aserin, A.; Garti, N. Maillard Reaction between Leucine and Glucose in $\mathrm{O} / \mathrm{W}$ Microemulsion Media in Comparison to Aqueous Solution. J. Dispersion Sci. Technol. 2005, 26, 535-547.

(26) Huang, R. D.; Feather, M. S. 13C NMR Study of Some Maillard Reaction Products Arising from D-Glucose-DL-Alanine Interactions. J. Agric. Food Chem. 1988, 36, 673-676.

(27) Tressl, R.; Wondrak, G. T.; Garbe, L. A.; Krüger, R. P.; Rewicki, D. Pentoses and Hexoses as Sources of New Melanoidin-like Maillard Polymers. J. Agric. Food Chem. 1998, 46, 1765-1776.

(28) Limacher, A.; Kerler, J.; Davidek, T.; Schmalzried, F.; Blank, I. Formation of Furan and Methylfuran by Maillard-Type Reactions in Model Systems and Food. J. Agric. Food Chem. 2008, 56, 3639-3647.

(29) Yaylayan, V. A.; Keyhani, A. Origin of Carbohydrate Degradation Products in L-Alanine/D- [13C]Glucose Model Systems. J. Agric. Food Chem. 2000, 48, 2415-2419.

(30) Yaylayan, V. A.; Keyhani, A. Origin of 2,3-Pentanedione and 2,3-Butanedione in D-Glucose/L-Alanine Maillard Model Systems. J. Agric. Food Chem. 1999, 47, 3280-3284.

(31) Ajandouz, E. H.; Puigserver, A. Nonenzymatic Browning Reaction of Essential Amino Acids: Effect of PH on Caramelization and Maillard Reaction Kinetics. J. Agric. Food Chem. 1999, 47, 17861793.

(32) Berton, C.; Ropers, M. H.; Viau, M.; Genot, C. Contribution of the Interfacial Layer to the Protection of Emulsified Lipids against Oxidation. J. Agric. Food Chem. 2011, 59, 5052-5061.

(33) Hsu, J. P.; Nacu, A. Behavior of Soybean Oil-in-Water Emulsion Stabilized by Nonionic Surfactant. J. Colloid Interface Sci. 2003, 259, 374-381. 
(34) Davídek, T.; Robert, F.; Devaud, S.; Vera, F. A.; Blank, I. Sugar Fragmentation in the Maillard Reaction Cascade: Formation of ShortChain Carboxylic Acids by a New Oxidative $\alpha$-Dicarbonyl Cleavage Pathway. J. Agric. Food Chem. 2006, 54, 6677-6684.

(35) Martins, S. I. F. S.; van Boekel, M. A. J. S. Kinetics of the Glucose/Glycine Maillard Reaction Pathways: Influences of $\mathrm{PH}$ and Reactant Initial Concentrations. Food Chem. 2005, 92, 437-448.

(36) Kim, J. S.; Lee, Y. S. Enolization and Racemization Reactions of Glucose and Fructose on Heating with Amino-Acid Enantiomers and the Formation of Melanoidins as a Result of the Maillard Reaction. Amino Acids 2009, 36, 465-474.

(37) Häger, M.; Currie, F.; Holmberg, K. A Nucleophilic Substitution Reaction in Microemulsions Based on Either an Alcohol Ethoxylate or a Sugar Surfactant. Colloids Surf., A 2004, 250, 163170.

(38) Yaghmur, A.; Aserin, A.; Abbas, A.; Garti, N. Reactivity of Furfural-Cysteine Model Reaction in Food-Grade Five-Component Nonionic O/W Microemulsions. Colloids Surf., A 2005, 253, 223234.

(39) Smuda, M.; Glomb, M. A. Fragmentation Pathways during Maillard-Induced Carbohydrate Degradation. In J. Agric. Food Chem. 2013 (61) 43, 10198-10208, DOI: 10.1021/jf305117s.

(40) Cerny, C.; Guntz-Dubini, R. Role of the Solvent Glycerol in the Maillard Reaction of D-Fructose and L-Alanine. J. Agric. Food Chem. 2006, 54, 574-577.

(41) Suratkar, V.; Mahapatra, S. Solubilization Site of Organic Perfume Molecules in Sodium Dodecyl Sulfate Micelles: New Insights from Proton NMR Studies. J. Colloid Interface Sci. 2000, 225, 32-38.

(42) Ottinger, H.; Hofmann, T. Identification of the Taste Enhancer Alapyridaine in Beef Broth and Evaluation of its Sensory Impact by Taste Reconstitution Experiments. J. Agric. Food Chem. 2003, 51, 6791-6796. 